\title{
The Effect of Inclination into Heat Convection Flow of Dissipative Fluid in a Channel of Porous walls
}

\author{
Tagread H. Shuker \\ Department of Mathematics \\ College of Education \\ University of Mosul, Iraq
}

Received on: 27/02/2013

Accepted on: 03/04/2013

\section{ABSTRACT}

This paper is devoted to the study of the effect of inclination into heat transfer by natural convection of fluid flowing through a channel with porous walls; the model has been created by using two-dimensional Cartesian co-ordinate and the resulting differential equations governing the model solved numerically in two phases (steadystate and unsteady-state) based on finite difference method.

Keywords: inclination, heat convection, dissipative fluid, porous walls.

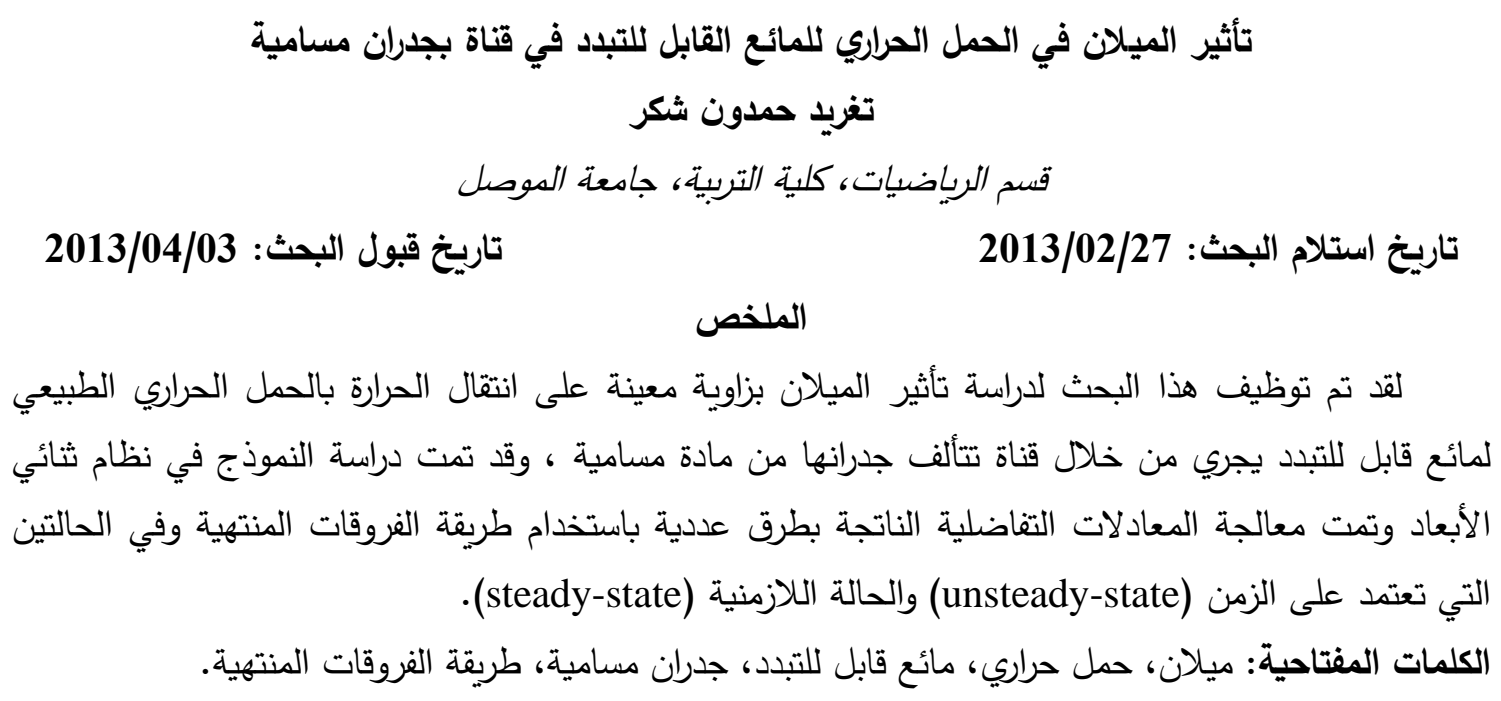

\section{Introduction}

Heat transfer is one of a very important subject for which has taken a wide intension of researchers and scientists and this is because of its applications specially after the Second world war, heat transfer by convection in a porous medium has many implementations in our life and this is due to the fact that the study of fluid flow in channels of porous walls has a relationship with blood flows inside the animals and human beings, solar porous collectors, geothermal energy systems, glass blowing etc ...

Many papers related to this matter have been written, Dlia S. C. [4], has studied the entropy generation production for a problem of a mixed convection flow of a fluid saturated porous medium through an inclined channel with uniform heated walls.

He used the analytical results obtained for the velocity and temperature profiles to obtain the entropy production, Jer-Huan Jang, Han-chich Chiu and Wei-Man Yan [6] gave a series of solutions for the laminar two-dimensional flow between two parallel porous plan walls driven by uniform injection and suction, they concluded that both fRe and $\mathrm{Nu}$ are increased with increasing mixed convection parameter through out the channel in the UHF (uniform walls temperature) case. But in the UWT( uniform walls temperature) case, the augmentation $\mathrm{FRe}$ and $\mathrm{Nu}$ are significant in the entrance region, C.I. Cooky, V.B. Omubo-Pepple [3] investigated the combined effects of radiative heat transfer and a transverse magnetic field on steady flow of an electrically conducting 
optically thin fluid through a horizontal channel filled with porous medium and nonuniform temperature at walls, they concluded that increasing magnetic field, radiation and porosity parameters reduced the velocity and temperature profiles as well as the share stress at the wall white increasing radiation parameters causes an increase in the magnitude of the rate of heat transfer, Gitima p. , Dusmanta K.S. [5] studied the effect of radiation on unsteady boundary layer flow with variable viscosity and thermal conductivity due to stretching sheet through porous channel in presence of magnetic field, numerical results using the shooting method are presented in terms of Darcy number parameter, they noticed that the values of Nasselt number increase with the increasing of radiation parameter and thermal conductivity parameter, while the skin friction has the opposite behavior, J.C. Umavathi [9] studied the flow and heat transfer in a vertical wavy channel, containing porous layer saturated with a fluid and clear viscous fluid layer, the Darcy-Brinkman model was adopted to describe the fluid flow, O.Anwar Beg [1] investigated the unsteady buoyancy-induced, hydromagnetic thermal convection flow in a semi-infinite porous region adjacent to an infinite hot vertical plate moving with constant velocity, the momentum and energy conservation equation are normalized and then solved by using both the Laplace transform technique and Network numerical simulation, G. Sudershan [8] has discussed the effect of radiation and chemical reaction on free convection MHD flow through a porous medium bounded by vertical surface. The governing equations of motion are solved analytically by using perturbation technique, Selamat M.S.[7] studied the natural convection in a porous cavity with a non-uniform hot wall temperature and a uniform cold wall temperature.

This work is devoted to the study of the inclined effect in the heat transfer by natural convection in a channel bounded by a permeable walls, the governing equations are solved by using a finite difference method under two cases, the steady and unsteady states.

\section{The Model and Governing Equations:}

Consider the unsteady flow of a dissipative fluid passing through a long channel with porous walls, inclined by the angle $\gamma$. The Cartesian coordinate system ( $x, y, z)$ has been taken as the ${ }^{x}$-axis lay in the center of the channel, ${ }^{y}$-axis represents the width of the channel while the $z$-axis is the normal of $x y$ plane. Let $u, v$ and $w$ be the velocity components in the directions $x, y$ and $z$ respectively; we assumed that all the components in $z$ direction are vanished as it,s illustrated by the Figure (1).

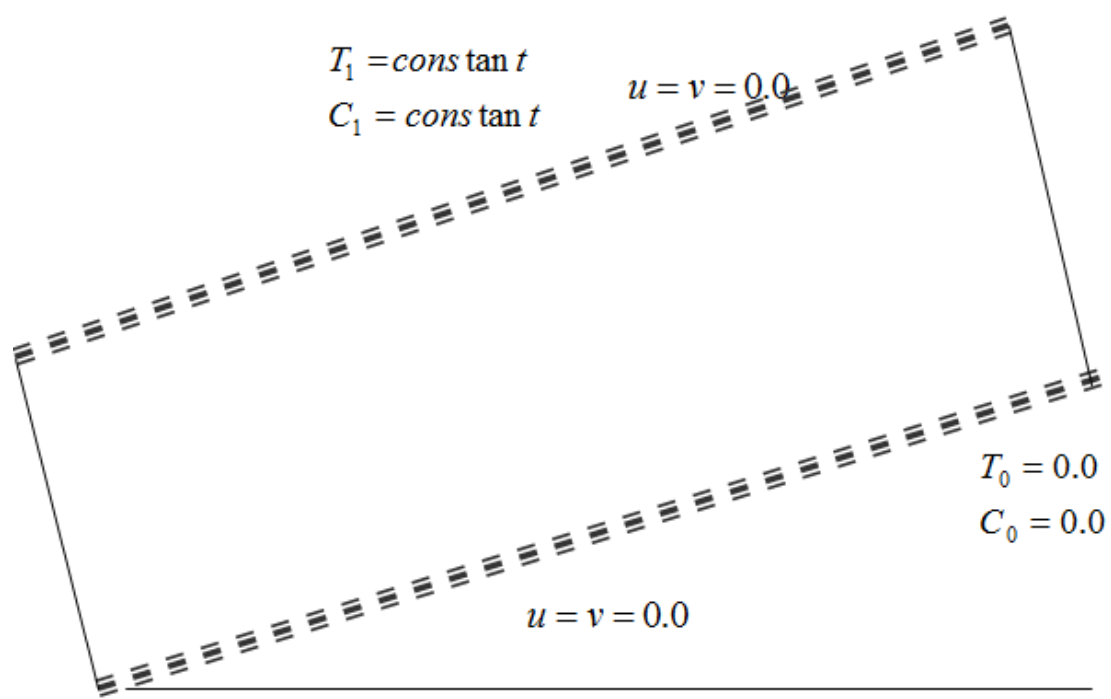


The governing equations in dimensional form are given by:

$$
\begin{aligned}
& \frac{\partial u}{\partial x}+\frac{\partial v}{\partial y}=0 \\
& \frac{\partial u}{\partial t}+u \frac{\partial u}{\partial x}+v \frac{\partial u}{\partial y}=\vartheta \nabla^{2} u+g \cos \gamma \beta\left(T-T_{1}\right)+g \cos \gamma \beta^{*}\left(C-C_{1}\right)-\frac{\vartheta}{k} u \\
& \frac{\partial T}{\partial t}+u \frac{\partial T}{\partial x}+v \frac{\partial T}{\partial y}=\alpha \nabla^{2} T+\frac{\mu}{\rho c_{p}}\left[\left(\frac{\partial u}{\partial y}\right)^{2}\right] \\
& \frac{\partial C}{\partial t}+u \frac{\partial C}{\partial x}+v \frac{\partial C}{\partial y}=D \nabla^{2} C
\end{aligned}
$$

Where $u, v$ are the velocity components, $t$ is the time and $T, g, \beta, \beta^{*}, \vartheta, k, \alpha, \mu, \rho, c_{p}, D, \mathrm{C}$ are the temperature, gravitational acceleration, thermal expansion coefficient, concentration expansion coefficient, Kinematics viscosity, permeability of the medium, thermal diffusivity, dynamical viscosity, density, specific heat at constant pressure, mass diffusion coefficient, concentration respectively.

With the following boundary conditions,

$$
\left.\begin{array}{l}
u=v=0.0 \\
T=T_{0}, T_{1} \\
C=C_{0}, C_{1}
\end{array}\right\} \quad y=0, h
$$

$\mathrm{h}$ is the width of the channel.

\section{Non-Dimensional Form:}

To solve the governing equations (2.1)-( 2.4) with the boundary conditions (2.5); we need to introduce the following non-dimensional quantities [4 and 3],

$$
\left.\begin{array}{l}
X=\frac{x}{h}, \quad Y=\frac{y G r^{1 / 4}}{h}, \quad U=\frac{u h(G r)^{-1 / 2}}{\vartheta}, \quad V=\frac{v h(G r)^{-1 / 4}}{\vartheta} \\
\tau=\frac{t \vartheta G r^{1 / 2}}{h^{2}}, \quad p r=\frac{\vartheta}{\alpha}, \quad \theta=\frac{T-T_{0}}{T_{0}-T_{1}}, \quad \phi=\frac{C-C_{1}}{C_{0}-C_{1}} \\
G r=\frac{g \beta h^{3}\left(T_{0}-T_{1}\right)}{\vartheta^{2}}, \quad G r^{*}=\frac{g \beta^{*} h^{3}\left(C_{0}-C_{1}\right)}{\vartheta^{2}}
\end{array}\right\}
$$

Substituting these quantities into equations (2.1)-(2.4), the governing equations become.

$$
\left.\begin{array}{l}
{\left[\begin{array}{l}
\sqrt{G r} \vartheta \\
h^{2}
\end{array}\right] \frac{\partial U}{\partial X}+\left[\frac{\sqrt{G r} \vartheta}{h^{2}}\right] \frac{\partial V}{\partial Y}=0} \\
{\left[\frac{G r \vartheta^{2}}{h^{3}}\right] \frac{\partial U}{\partial \tau}+\left[\frac{G r \vartheta^{2}}{h^{3}}\right] U \frac{\partial U}{\partial X}+\left[\frac{G r \vartheta^{2}}{h^{3}}\right] V \frac{\partial U}{\partial Y}=\left[\frac{\sqrt{G r} \vartheta^{2}}{h^{3}}\right] \frac{\partial^{2} U}{\partial X^{2}}+\left[\frac{G r \vartheta^{2}}{h^{3}}\right] \frac{\partial^{2} U}{\partial Y^{2}}+} \\
+g \cos \gamma \beta\left(T_{0}-T_{1}\right)+g \cos \gamma \beta^{*}\left(C_{0}-C_{1}\right)-\frac{\sqrt{G r} \vartheta^{2}}{K h} U
\end{array}\right]
$$




$$
\begin{gathered}
{\left[\frac{\left(T_{0}-T_{1}\right) \sqrt{G r} \vartheta}{h^{2}}\right] \frac{\partial \theta}{\partial \tau}+\left[\frac{\left(T_{0}-T_{1}\right) \sqrt{G r} \vartheta}{h^{2}}\right] U \frac{\partial \theta}{\partial X}+\left[\frac{\left(T_{0}-T_{1}\right) \sqrt{G r} \vartheta}{h^{2}}\right] V \frac{\partial \theta}{\partial Y}=} \\
=\left[\frac{\alpha\left(T_{0}-T_{1}\right)}{h^{2}}\right] \frac{\partial^{2} \theta}{\partial X^{2}}+\left[\frac{\alpha\left(T_{0}-T_{1}\right) \sqrt{G r}}{h^{2}}\right] \frac{\partial^{2} \theta}{\partial Y^{2}}+\left(\frac{\mu}{\rho c_{p}}\right)\left[\frac{G r \sqrt{G r} \vartheta^{2}}{h^{4}}\right]\left(\frac{\partial U}{\partial Y}\right)^{2} \\
{\left[\frac{\left(C_{0}-C_{1}\right) \sqrt{G r} \vartheta}{h^{2}}\right] \frac{\partial \phi}{\partial \tau}+\left[\frac{\left(C_{0}-C_{1}\right) \sqrt{G r} \vartheta}{h^{2}}\right] U \frac{\partial \phi}{\partial X}\left[\frac{\left(C_{0}-C_{1}\right) \sqrt{G r} \vartheta}{h^{2}}\right] V \frac{\partial \phi}{\partial Y}=} \\
=D\left[\left(\frac{\left(C_{0}-C_{1}\right)}{h^{2}}\right) \frac{\partial^{2} \phi}{\partial X^{2}}+\left(\frac{\left(C_{0}-C_{1}\right) \sqrt{G r}}{h^{2}}\right) \frac{\partial^{2} \phi}{\partial Y^{2}}\right]
\end{gathered}
$$

Simplifying the above equations, the governing equations under these non-dimensional quantities become,

$$
\begin{aligned}
& \frac{\partial U}{\partial X}+\frac{\partial V}{\partial Y}=0 \\
& \frac{\partial U}{\partial \tau}+U \frac{\partial U}{\partial X}+V \frac{\partial U}{\partial Y}=\frac{1}{\sqrt{G r}} \frac{\partial^{2} U}{\partial X^{2}}+\frac{\partial^{2} U}{\partial Y^{2}}+\cos \gamma \theta+\frac{G r^{*}}{G r} \cos \gamma \phi-\frac{1}{\sqrt{G r} D a} U \\
& \frac{\partial \theta}{\partial \tau}+U \frac{\partial \theta}{\partial X}+V \frac{\partial \theta}{\partial Y}=\frac{1}{\sqrt{G r} p r} \frac{\partial^{2} \theta}{\partial X^{2}}+\frac{1}{p r} \frac{\partial^{2} \theta}{\partial Y^{2}}+\varepsilon\left(\frac{\partial U}{\partial Y}\right)^{2} \\
& \frac{\partial \phi}{\partial \tau}+U \frac{\partial \phi}{\partial X}+V \frac{\partial \phi}{\partial Y}=\frac{1}{S c}\left[\frac{1}{\sqrt{G r}} \frac{\partial^{2} \phi}{\partial X^{2}}+\frac{\partial^{2} \phi}{\partial Y^{2}}\right]
\end{aligned}
$$

where

$G r \quad=$ Grashof number for heat transfer

$\varepsilon=\frac{g \beta h}{C_{p}}=$ dissipation parameter

pr $\quad=$ Prandtl number

$G r^{*} \quad=$ Grashof number for Mass transfer

$S c=\frac{\vartheta}{D}=$ Schmidt number

$\mathrm{D}=$ the mass diffusion coefficient

$$
\mathrm{Da}=\frac{K}{h^{2}}=\text { Darcy number }
$$

and the boundary conditions (2.5) in the non-dimensional form become,

$$
\left.\begin{array}{lrlrl}
U & =V=0 & & \text { at } Y=0,1 \\
\theta & =0.0 & & \text { at } Y=0 \\
\theta & =10.0 & & \text { at } Y=1 \\
\phi & =0.0 & & \text { at } Y=0 \\
\phi & =1.0 & & \text { at } Y=1
\end{array}\right\}
$$


Where, $\phi$ and $\theta$ are taken above for the simplicity of calculation.

\section{4- Method of Solution:}

In order to solve the system of equations (3.2)-(3.5) with the boundary conditions (3.6); we resort to ADI finite difference method [4], and to achieve this, we have to start with the last equation (3.5), equation of diffusion and then equation (3.4), heat equation, and finally equation (3.3), equation of motion as following:

\section{4-1 Solution of Diffusion Equation:}

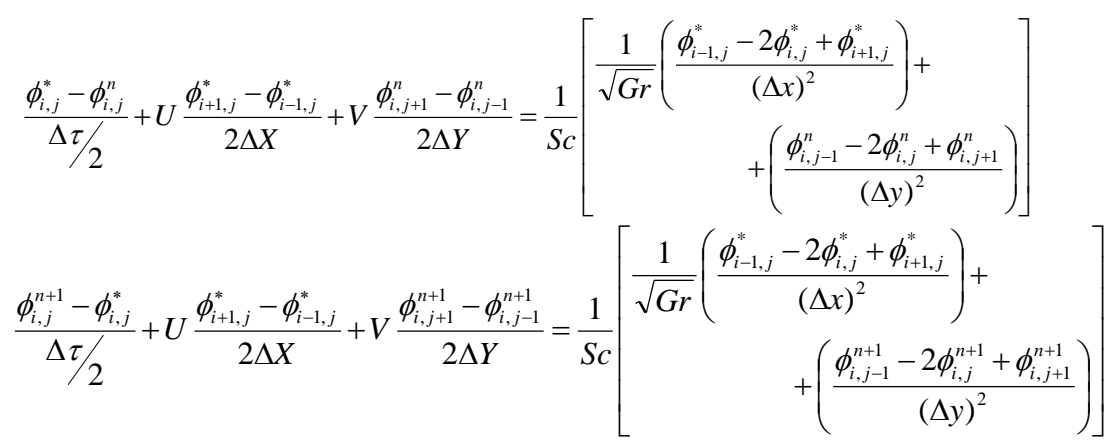

with boundary conditions,

$\left.\begin{array}{lll}U=\text { cons } \tan t & U_{0, j}^{n}=0, & U_{i, 0}^{n}=0 \\ V=\text { cons } \tan t & V_{0, j}^{n}=0, & V_{i, 0}^{n}=0 \\ \phi_{i, 0}^{n}=0.0 & \phi_{i, N}^{n}=1.0 & \end{array}\right\}$

Equations (4.1.1) and (4.1.2) can be reduced to give,

$$
A(I) \phi_{i-1, j}^{*}+B(I) \phi_{i, j}^{*}+C(I) \phi_{i+1, j}^{*}=D_{1}(I), \quad I=0,1,2, \ldots N
$$

where

$$
\left.\begin{array}{l}
A(I)=-\left(U \frac{\Delta \tau}{2 \Delta X}+\frac{\Delta \tau}{S c \sqrt{G r}(\Delta X)^{2}}\right) \\
B(I)=2\left(1+\frac{\Delta \tau}{S c \sqrt{G r}(\Delta X)^{2}}\right) \\
C(I)=\frac{U \Delta \tau}{2 \Delta X}-\frac{\Delta \tau}{S c \sqrt{G r}(\Delta X)^{2}} \\
D_{1}(I)=\left(\frac{V \Delta \tau}{2 \Delta y}+\frac{\Delta \tau}{S c(\Delta Y)^{2}}\right) \phi_{i, j-1}^{n}+2\left(1-\frac{\Delta \tau}{S c(\Delta Y)^{2}}\right) \phi_{i, j}^{n}+\left(\frac{\Delta \tau}{S c(\Delta Y)^{2}}-\frac{V \Delta \tau}{2 \Delta Y}\right) \phi_{i, j+1}^{n}
\end{array}\right\}
$$

followed by,

$$
A_{1}(J) \phi_{i, j-1}^{n+1}+B_{1}(J) \phi_{i, j}^{n+1}+C_{1}(J) \phi_{i, j+1}^{n+1}=D_{2}(J), \quad J=0,1,2, \ldots N
$$

where 


$$
\left.\begin{array}{rl}
A_{1}(J) & =-\left(\frac{\Delta \tau}{S c \sqrt{G r}(\Delta Y)^{2}}+V \frac{\Delta \tau}{2 \Delta Y}\right) \\
B_{1}(J) & =2\left(1+\frac{\Delta \tau}{S c(\Delta Y)^{2}}\right) \\
C_{1}(J) & =\frac{V \Delta \tau}{2 \Delta Y}-\frac{\Delta \tau}{S c(\Delta Y)^{2}} \\
D_{2}(J) & =\left(\frac{U \Delta \tau}{2 \Delta X}+\frac{\Delta \tau}{S c \sqrt{G r}(\Delta X)^{2}}\right) \phi_{i-1, j}^{*}+2\left(1-\frac{\Delta \tau}{S c \sqrt{G r}(\Delta X)^{2}}\right) \phi_{i, j}^{*}+ \\
& \quad+\left(\frac{\Delta \tau}{S c \sqrt{G r}(\Delta X)^{2}}-\frac{U \Delta \tau}{2 \Delta X}\right) \phi_{i+1, j}^{*}
\end{array}\right\}
$$

\section{4-2 Solution of Heat Equation:}

$$
\begin{aligned}
& \frac{\theta_{i, j}^{*}-\theta_{i, j}^{n}}{\Delta \tau / 2}+U \frac{\theta_{i+1, j}^{*}-\theta_{i-1, j}^{*}}{2 \Delta X}+V \frac{\theta_{i, j+1}^{n}-\theta_{i, j-1}^{n}}{2 \Delta Y}= \frac{1}{p r \sqrt{G r}}\left(\frac{\theta_{i-1, j}^{*}-2 \theta_{i, j}^{*}+\theta_{i+1, j}^{*}}{(\Delta x)^{2}}\right)+ \\
&+\frac{1}{p r}\left(\frac{\theta_{i, j-1}^{n}-2 \theta_{i, j}^{n}+\theta_{i, j+1}^{n}}{(\Delta y)^{2}}\right)+\varepsilon\left(\frac{U}{\Delta Y}\right)^{2} \\
& \frac{\theta_{i, j}^{n+1}-\theta_{i, j}^{*}}{\Delta \tau / 2}+U \frac{\theta_{i+1, j}^{*}-\theta_{i-1, j}^{*}}{2 \Delta X}+V \frac{\theta_{i, j+1}^{n+1}-\theta_{i, j-1}^{n+1}}{2 \Delta Y}=\frac{1}{p r \sqrt{G r}}\left(\frac{\theta_{i-1, j}^{*}-2 \theta_{i, j}^{*}+\theta_{i+1, j}^{*}}{(\Delta x)^{2}}\right)+ \\
&+\frac{1}{p r}\left(\frac{\theta_{i, j-1}^{n+1}-2 \theta_{i, j}^{n+1}+\theta_{i, j+1}^{n+1}}{(\Delta y)^{2}}\right)+\varepsilon\left(\frac{U}{\Delta Y}\right)^{2}
\end{aligned}
$$

with boundary conditions,

$$
\left.\begin{array}{lll}
U=\text { cons } \tan t & U_{0, j}^{n}=0, & U_{i, 0}^{n}=0 \\
V=\text { cons } \tan t & V_{0, j}^{n}=0, & V_{i, 0}^{n}=0 \\
\theta_{i, 0}^{n}=0.0 & \theta_{i, N}^{n}=10.0
\end{array}\right\}
$$

Equations (4.2.1) and (4.2.2) can be reduced to give,

$$
A_{2}(I) \theta_{i-1, j}^{*}+B_{2}(I) \theta_{i, j}^{*}+C_{2}(I) \theta_{i+1, j}^{*}=D_{3}(I), \quad I=0,1,2, \ldots N
$$

Where,

$$
\left.\begin{array}{rl}
A_{2}(I) & =-\left(U \frac{\Delta \tau}{2 \Delta X}+\frac{\Delta \tau}{p r \sqrt{G r}(\Delta X)^{2}}\right) \\
B_{2}(I) & =2\left(1+\frac{\Delta \tau}{p r \sqrt{G r}(\Delta X)^{2}}\right) \\
C_{2}(I) & =\frac{U \Delta \tau}{2 \Delta X}-\frac{\Delta \tau}{p r \sqrt{G r}(\Delta X)^{2}} \\
D_{3}(I) & =\left(\frac{V \Delta \tau}{2 \Delta y}+\frac{\Delta \tau}{p r(\Delta Y)^{2}}\right) \theta_{i, j-1}^{n}+2\left(1-\frac{\Delta \tau}{p r(\Delta Y)^{2}}\right) \theta_{i, j}^{n}+\left(\frac{\Delta \tau}{p r(\Delta Y)^{2}}-\frac{V \Delta \tau}{2 \Delta Y}\right) \theta_{i, j+1}^{n}+ \\
& +\varepsilon\left(\frac{U}{\Delta Y}\right)^{2}
\end{array}\right\}
$$


followed by,

$$
A_{3}(J) \theta_{i, j-1}^{n+1}+B_{3}(J) \theta_{i, j}^{n+1}+C_{3}(J) \theta_{i, j+1}^{n+1}=D_{4}(J), \quad J=0,1,2, \ldots N
$$

where

$$
\left.\begin{array}{rl}
A_{3}(J) & =-\left(\frac{\Delta \tau}{p r(\Delta Y)^{2}}+V \frac{\Delta \tau}{2 \Delta Y}\right) \\
B_{3}(J) & =2\left(1+\frac{\Delta \tau}{p r(\Delta Y)^{2}}\right) \\
C_{3}(J) & =\frac{V \Delta \tau}{2 \Delta Y}-\frac{\Delta \tau}{p r(\Delta Y)^{2}} \\
D_{4}(J) & =\left(\frac{U \Delta \tau}{2 \Delta X}+\frac{\Delta \tau}{p r \sqrt{G r}(\Delta X)^{2}}\right) \theta_{i-1, j}^{*}+2\left(1-\frac{\Delta \tau}{p r \sqrt{G r}(\Delta X)^{2}}\right) \theta_{i, j}^{*}+ \\
& +\left(\frac{\Delta \tau}{p r \sqrt{G r}(\Delta X)^{2}}-\frac{U \Delta \tau}{2 \Delta X}\right) \theta_{i+1, j}^{*}+\varepsilon\left(\frac{U}{\Delta Y}\right)^{2}
\end{array}\right\}
$$

\section{4-3 Solution Equation of Motion:}

$$
\begin{gathered}
\frac{U_{i, j}^{*}-U_{i, j}^{n}}{\Delta \tau / 2}+U \frac{U_{i+1, j}^{*}-U_{i-1, j}^{*}}{2 \Delta X}+V \frac{U_{i, j+1}^{n}-U_{i, j-1}^{n}}{2 \Delta Y}=\frac{1}{\sqrt{G r}}\left(\frac{U_{i-1, j}^{*}-2 U_{i, j}^{*}+U_{i+1, j}^{*}}{(\Delta x)^{2}}\right)+ \\
+\left(\frac{U_{i, j-1}^{n}-2 U_{i, j}^{n}+U_{i, j+1}^{n}}{(\Delta y)^{2}}\right)+\cos \gamma \theta_{i, j}^{n}+\frac{G r^{*}}{G r} \cos \gamma \phi_{i, j}^{n}-\frac{1}{\sqrt{G r} D a} U \\
\frac{U_{i, j}^{n+1}-U_{i, j}^{*}}{\Delta \tau / 2}+U \frac{U_{i+1, j}^{*}-U_{i-1, j}^{*}}{2 \Delta X}+V \frac{U_{i, j+1}^{n+1}-U_{i, j-1}^{n+1}}{2 \Delta Y}=\frac{1}{\sqrt{G r}}\left(\frac{U_{i-1, j}^{*}-2 U_{i, j}^{*}+U_{i+1, j}^{*}}{(\Delta x)^{2}}\right)+ \\
+\left(\frac{U_{i, j-1}^{n+1}-2 U_{i, j}^{n+1}+U_{i, j+1}^{n+1}}{(\Delta y)^{2}}\right)+\cos \gamma \theta_{i, j}^{n+1}+\frac{G r^{*}}{G r} \cos \gamma \phi_{i, j}^{n+1}-\frac{1}{\sqrt{G r} D a} U
\end{gathered}
$$

with boundary conditions,

$$
\left.\begin{array}{ccc}
U=\text { cons } \tan t & U_{0, j}^{n}=0, & U_{i, 0}^{n}=0 \\
V=\text { cons } \tan t & V_{0, j}^{n}=0, & V_{i, 0}^{n}=0
\end{array}\right\}
$$

Equations (4.3.1) and (4.3.2) can be reduced to give, (see [2] ).

$$
A_{4}(I) U_{i-1, j}^{*}+B_{4}(I) U_{i, j}^{*}+C_{4}(I) U_{i+1, j}^{*}=D_{5}(I), \quad I=0,1,2, \ldots N
$$

Where, 


$$
\begin{aligned}
A_{4}(I)= & -\left(U \frac{\Delta \tau}{2 \Delta X}+\frac{\Delta \tau}{\sqrt{G r}(\Delta X)^{2}}\right) \\
B_{4}(I)=2\left(1+\frac{\Delta \tau}{\sqrt{G r}(\Delta X)^{2}}\right) & \left.\begin{array}{rl}
C_{4}(I)= & \frac{U \Delta \tau}{2 \Delta X}-\frac{\Delta \tau}{\sqrt{G r}(\Delta X)^{2}} \\
D_{5}(I)=\left(\frac{V \Delta \tau}{2 \Delta y}+\frac{\Delta \tau}{(\Delta Y)^{2}}\right) U_{i, j-1}^{n} & +2\left(1-\frac{\Delta \tau}{(\Delta Y)^{2}}\right) U_{i, j}^{n}+\left(\frac{\Delta \tau}{(\Delta Y)^{2}}-\frac{V \Delta \tau}{2 \Delta Y}\right) U_{i, j+1}^{n}+ \\
& +\Delta \tau \cos \gamma \theta_{i, j}^{n+1}+\frac{\Delta \tau G r^{*}}{G r} \cos \gamma \phi_{i, j}^{n+1}-\frac{\Delta \tau}{\sqrt{G r} D a} U
\end{array}\right\}
\end{aligned}
$$

followed by,

$$
A_{5}(J) U_{i, j-1}^{n+1}+B_{5}(J) U_{i, j}^{n+1}+C_{5}(J) U_{i, j+1}^{n+1}=D_{6}(J), \quad J=0,1,2, \ldots N
$$

where

$$
\left.\begin{array}{rl}
A_{5}(J)= & -\left(\frac{\Delta \tau}{(\Delta Y)^{2}}+V \frac{\Delta \tau}{2 \Delta Y}\right) \\
B_{5}(J)=2\left(1+\frac{\Delta \tau}{(\Delta Y)^{2}}\right) & \\
C_{5}(J)= & \frac{V \Delta \tau}{2 \Delta Y}-\frac{\Delta \tau}{(\Delta Y)^{2}} \\
D_{6}(J)= & \left(\frac{U \Delta \tau}{2 \Delta X}+\frac{\Delta \tau}{\sqrt{G r}(\Delta X)^{2}}\right) U_{i-1, j}^{*}+2\left(1-\frac{\Delta \tau}{\sqrt{G r}(\Delta X)^{2}}\right) U_{i, j}^{*}+ \\
& +\left(\frac{\Delta \tau}{\sqrt{G r}(\Delta X)^{2}}-\frac{U \Delta \tau}{2 \Delta X}\right) U_{i+1, j}^{*}+\Delta \tau \cos \gamma \theta_{i, j}^{n+1}+\frac{\Delta \tau G r^{*}}{G r} \cos \gamma \phi_{i, j}^{n+1}-\frac{\Delta \tau}{\sqrt{G r} D a} U
\end{array}\right\}
$$

The coefficients $U, V$ are treated as constants during any one time-step of the computation [5], each of the equations (diffusion, heat, motion) creating a tridiagonal system which are solved by using Gauss elimination method, all details are given in. [4].

\section{5- Conclusions:}

We present in this section some of the results obtained from the computation done on equation of motion (3.3) for different point in the region of solution and this is because of the appearance of inclination effect on this equation, these results have been expressed by figures to illustrate how the inclination angle play an important role in the behavior of the velocity function $u$ at the different places of the solution region. Figure (2) represents the curve of the velocity function at the lower part of the region for different values of some parameters and different value of angles as it is shown in the figures. The behavior of the velocity function $u$ at the middle of the region is given in Figure (3), finally, Figure (4) represents the the velocity function at the upper part.

It has been noticed also that some parameters like ( Grashof number Gr, and Schmidt number Sc) played a significant role in the solution of the problem , 


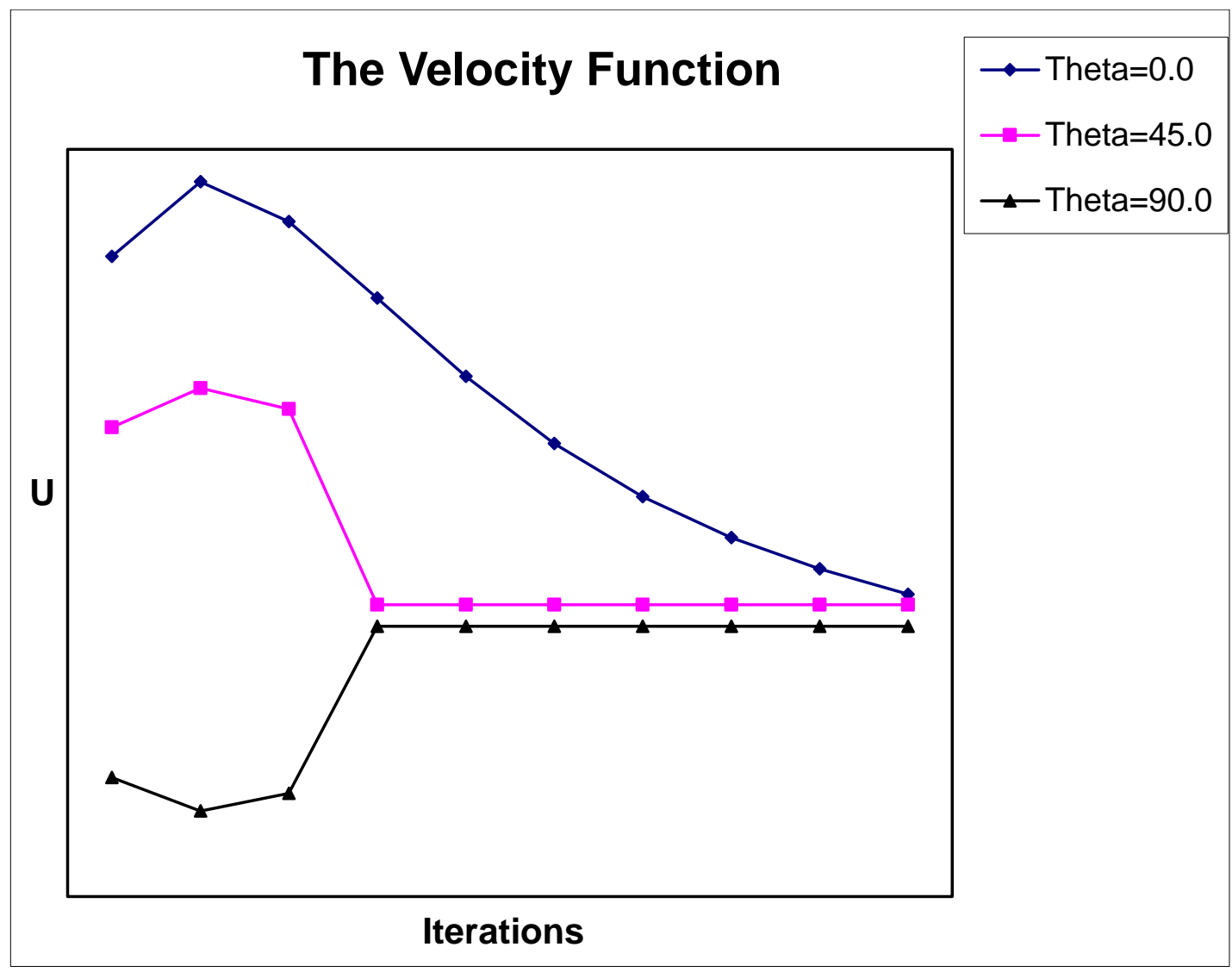

Figure (2) The non-dimensional velocity function $u$ for different value of the angle at the lower part of the region with the parameters: $G r=0.1, S c=0.22$

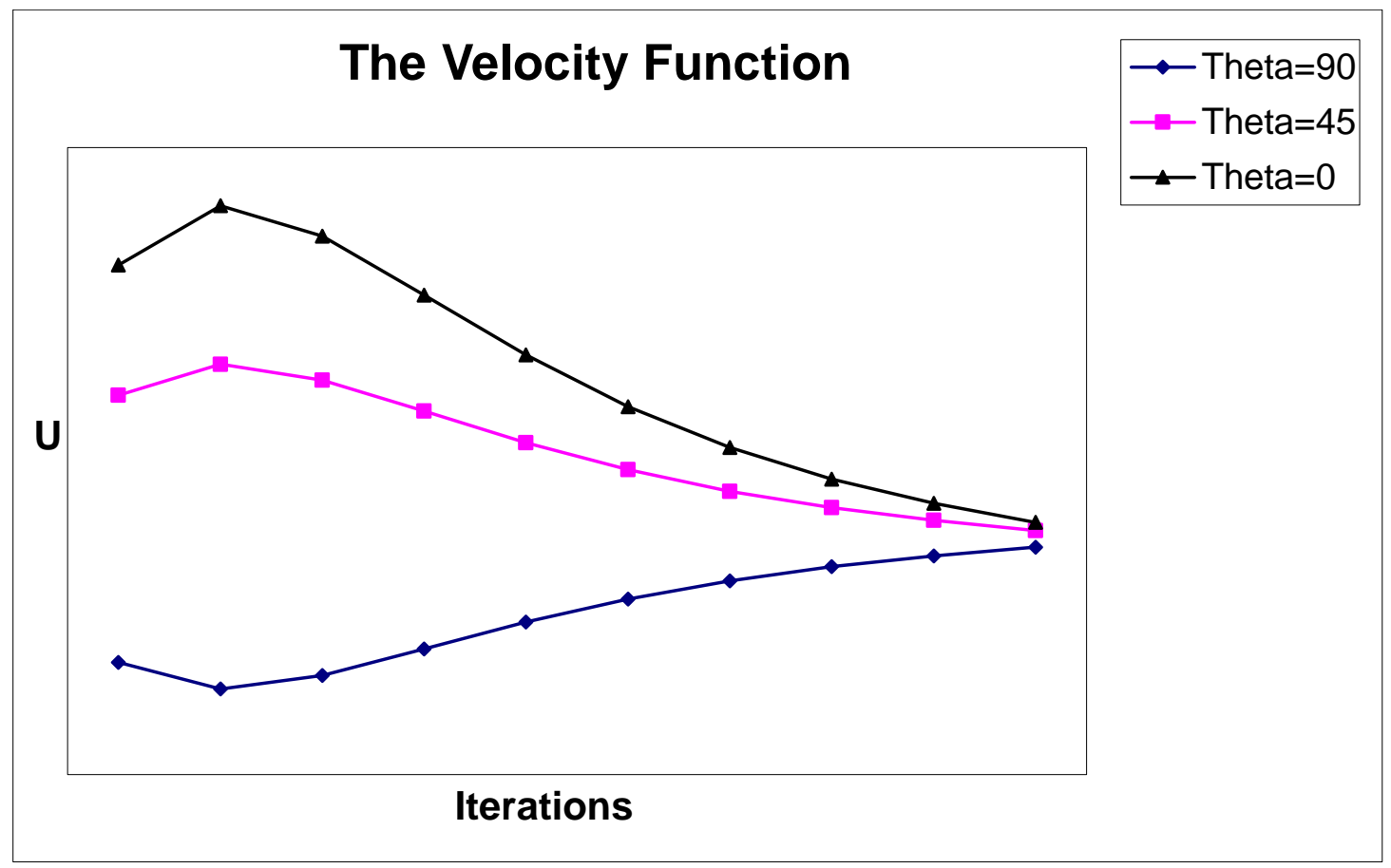

Figure (3) The non-dimensional velocity function $u$ for different value of the angle at the middle part of the region with the parameters: $G r=0.1, S c=0.22$ 


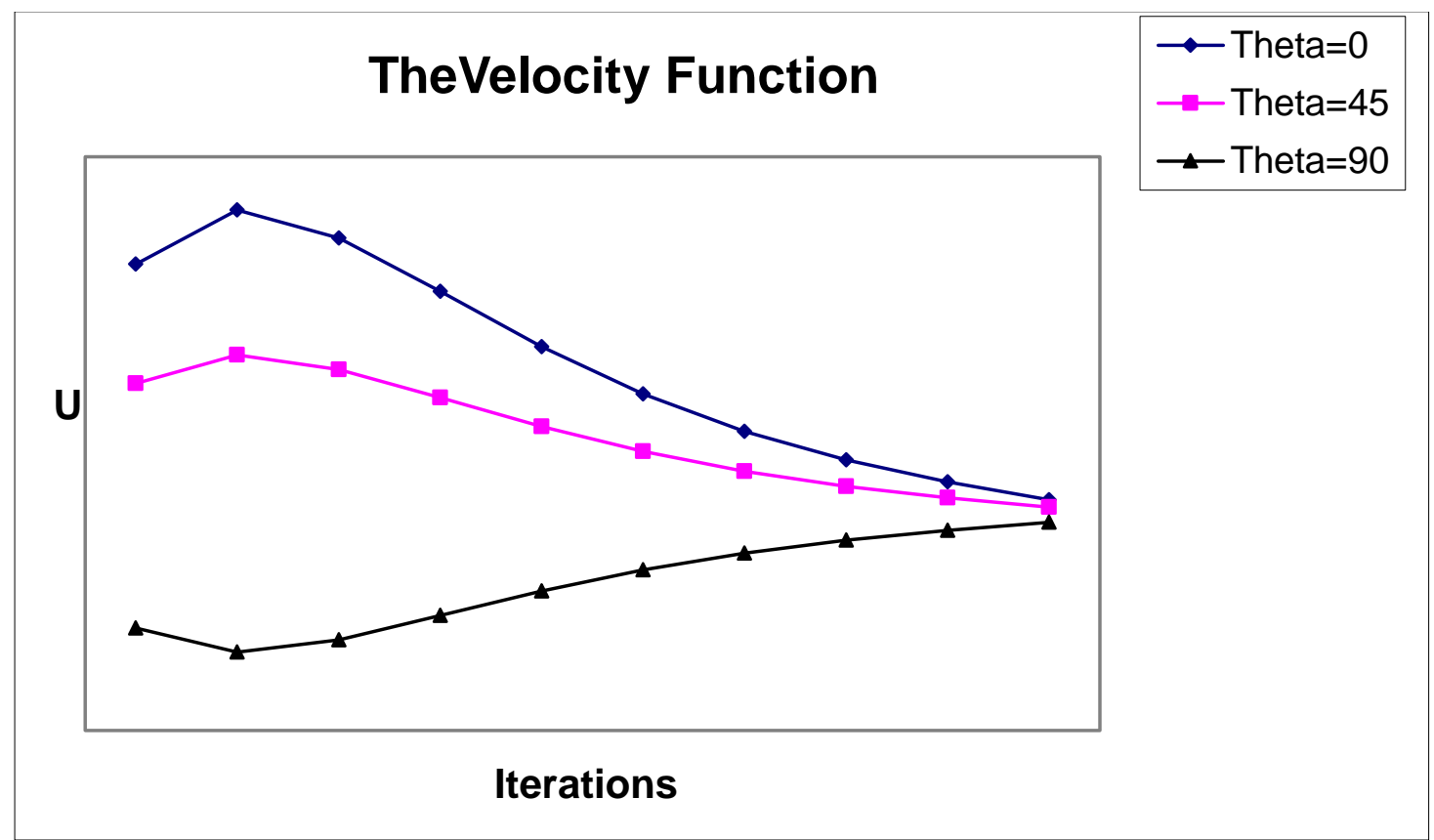

Figure (4) The non-dimensional velocity function $u$ for different value of the angle at the upper part of the region with the parameters: $G r=0.1, S c=0.22$. 


\section{REFERENCES}

[1] Anwar Beg O., Zueco J, Ghoosh S. K. and Alireza Heidari, 2011,"Unsteady Magnetohydrodynamic Heat transfer in a Semi-Infinite porous Medium with thermal Radiation flux: Analytical and Numerical study", Hindawi Publishing Corporation, Advances in Numerical Analysis . Vol 2011, Article ID 304124, 17 pages.

[2] Brice Carnhan, H.A. Luther, James O.Wilkes, 1969, " Applied Numerical Methods ", John Wiley \& Sons, INC. New York. London. Sydney. Toronto

[3] Cookey C.I. , V.B. Omubo-Pepple, , 2010, “On steady hydro genetic flow of a radiating viscous fluid through a horizontal channel in a porous media ", AMARECAN JOURNAL OF SCIENCE AND INDUSTRIAL RESEARCH, ISSN 2153-649xX, pp 203-208.

[4] Dalia S.C., Ioan P., 2011, "A study of entropy generation minimization in an inclined channel ", WSEAS TRANSACTIONS on HEAT and MASS TRANSFER, ISSN 1790-5044, Issue2, Volume 6.

[5] Gitima P., Dusmanta K. S., 2011, "Study the effect of variable viscosity and thermal conductivity of micropolar fluid in a porous channel", Dusmanta Kumar Sut et al , Int. J. Comp. Tech. Appl. ,Vol.2 (5), No.2, pp. 1247-1255.

[6] Jer-Huan J.., Han-Chich C. and Wei-Mon Y., 2010, "Wall transpiration effects developing mixed convection heat transfer in inclined rectangular ducts ", J. of Marine Science and Technology, Vol 18 , No. 2, pp. 249258 .

[7] Selamat S.M., Roslan R. and Hashim I., 2012, "Natural Convection in an Inclined porous Cavity with Spatial Sidewall Temperature Variations", Hindawi Publishing Corporation, Journal of Applied Mathematics, Vol 2012, Article ID pages.

[8] Sudershan S.G. Reddy R.V.G. and Reddy J. K., 2012, "Radiation and chemical reaction effects on free convection MHD flow through a porous medium bounded by vertical surface ", Pelugia Reserch Library , Advances in Applied Science Research, 3(3): 1603-1610.

[9] Umavathi J.C. and Shakar M.., 2011, "Mixed convection flow and heat transfer in a vertical wavy channel containing porous and fluid layer with traveling thermal waves ", International journal of engineering, Science and Technology, Vol. 3 , No. 6, pp 196-219. 